\title{
Threshold resummation for dark-matter production at the LHC
}

\author{
M. Krämer, ${ }^{a}$ A. Kulesza, ${ }^{b}$ A. Mück ${ }^{a}$ and R. Schürmann ${ }^{a, c}$ \\ ${ }^{a}$ Institut für Theoretische Teilchenphysik und Kosmologie, RWTH Aachen University, \\ D-52056 Aachen, Germany \\ ${ }^{b}$ Institute for Theoretical Physics, WWU Münster, \\ D-48149 Münster, Germany \\ ${ }^{c}$ Physik-Institut, Universität Zürich, \\ Wintherturerstrasse 190, CH-8057 Zürich, Switzerland \\ E-mail: mkraemer@physik.rwth-aachen.de, anna.kulesza@uni-muenster.de, \\ mueck@physik.rwth-aachen.de, robins@physik.uzh.ch
}

ABSTRACT: We derive precision predictions for the production of dark-matter particles recoiling against a jet with large transverse momentum at the LHC. The dark-matter fermions are described within a simplified model and couple to the Standard Model via a vector mediator. Our predictions for the mono-jet signature include the resummation of the leading and next-to-leading threshold logarithms. The corresponding matching coefficient is evaluated at NLO. The resummed result is matched to the fixed-order NLO cross section obtained from the MadGraph framework. We discuss numerical results for several benchmark scenarios at the LHC.

KEywords: Beyond Standard Model, Resummation

ARXIV EPRINT: 1903.06417 


\section{Contents}

1 Introduction 1

2 Dark-matter model 3

3 Partonic channels and kinematic considerations 4

4 Resummed cross section $\quad 5$

$\begin{array}{lll}5 & \text { Numerical results } & 11\end{array}$

$\begin{array}{llr}6 & \text { Conclusions } & 15\end{array}$

$\begin{array}{ll}\text { A LO cross sections } & 16\end{array}$

\section{Introduction}

The nature of dark matter remains one of the big unanswered questions in modern physics. Weakly interacting massive particles (WIMPs) are well-motivated dark matter candidates and are predicted by many extensions of the Standard Model (SM) [1]. One of the main goals of the Large Hadron Collider (LHC) is to test the WIMP paradigm, see e.g. ref. [2]. Many LHC searches for WIMP dark matter rely on the mono-X signature, where one would observe a SM particle recoiling against the missing transverse energy caused by WIMPs escaping the detector.

To interpret the LHC data, precise predictions for the dark-matter production cross section and for the corresponding SM backgrounds are essential. The main irreducible $\mathrm{SM}$ background is Z-boson production in association with jets, where the $\mathrm{Z}$ boson decays invisibly into neutrinos. A thorough discussion of the theoretical description of this darkmatter background can be found in ref. [3].

Predictions for the dark-matter signal depend on the concrete model under consideration. We employ the simplified-model approach, refs. [4, 5], and derive precision predictions for the process $p p \rightarrow \chi \bar{\chi}+$ jet in a simplified dark-matter model with a fermionic darkmatter particle $\chi$ and an s-channel vector mediator $Y$ which couples to SM quarks but not to leptons [6]. Hence, stringent bounds from resonance searches in the Drell-Yan process are avoided. The LHC has also placed bounds on the simplified model parameter space employing resonance searches in the di-jet final state [7-9].

Mono-X production of dark matter has already been investigated in simplified models at next-to-leading order (NLO) in QCD [10-17]. The simplified dark-matter model with a vector mediator has been analyzed at NLO including jet merging and parton-shower effects [13]. The model has been implemented in the MadGraph5_aMC@NLO-framework [18] 
using FeynRules $[19,20]$ to obtain NLO predictions in a fully automated way. The NLO corrections result in a moderate enhancement of the integrated cross section and the missing transverse momentum distribution, depending in detail on the mediator and dark-matter masses. Moreover, a sizeable reduction of the scale dependence of the cross section is reported improving the predictions from LO to NLO, as expected.

Given the current WIMP exclusion limits from the LHC $[7,8]$ and from direct-detection experiments, see e.g. ref. [21], future LHC searches will focus on large dark-matter and/or mediator masses. In this case, due to the rapid decrease of the parton luminosities as a function of the partonic center-of-mass energy, a significant part of the cross section can be attributed to the threshold region. In this region, the partonic center-of-mass energy is not much larger than the minimal energy needed to produce the invariant mass of the dark-matter pair and the recoiling jet at a given transverse momentum. As discussed in section 3, higher-order corrections close to threshold contain large logarithms because realemission contributions are restricted to the emission of soft and collinear partons due to the lack of available energy. Hence, it is desirable to take these logarithms into account beyond NLO.

In this work, we refine the theoretical prediction of ref. [13] for inclusive observables in the process $p p \rightarrow \chi \bar{\chi}+$ jet by resumming potentially large threshold logarithms up to next-to-leading logarithmic (NLL) accuracy. More specifically, we perform the resummation for the double-differential cross section with respect to the transverse momentum and the invariant mass of the dark-matter particles in the final state. To calculate the anomalous dimensions for the renormalization-group evolution of the soft and the jet functions as well as the soft and collinear NLO contributions to the matching coefficient, we use the strategy of regions [22]. The NLL expressions are identical to those presented in ref. [23] after adapting the color-factors. The corresponding matching coefficient is calculated at NLO in the strong coupling $\alpha_{s}$. We call these predictions NLL' to distinguish them from NLL predictions with a LO matching coefficient. The hard function, which only enters the matching coefficient, is obtained numerically from MadLoop within the MadGraph5_aMC@NLO-framework [18]. Combining these numerical one-loop results with the analytic results obtained using the strategy of regions provides a convenient and straightforward way to calculate NLL' resummed cross sections. Since the invariant mass of the dark-matter particles is not accessible experimentally, we integrate the resummed distribution over the invariant mass. The resummed predictions are matched to the full fixed-order NLO prediction obtained from the MadGraph5_aMC@NLO-framework [18].

The resummation of threshold logarithms has been achieved for closely related SM processes. In particular, threshold resummation in the direct QCD approach was derived to NLL accuracy for direct photon hadroproduction in refs. [24-28]. The resummed results in one-particle inclusive kinematics were later used to obtain approximate higher-order corrections for direct photon hadroproduction as well as for the production of a massive gauge boson at high transverse momentum [29-32]. A general discussion of threshold resummation for single inclusive cross sections at NLL and NNLL can be found in refs. [26, 33, 34]. For the hadroproduction of Higgs bosons at large transverse momentum, threshold resummation is known to NLL' accuracy [23]. Recently, a similar analysis for the closely 
related Drell-Yan process at a fixed invariant mass and high transverse momentum has been performed in ref. [35]. Within the formalism of Soft-Collinear-Effective-Field-Theory (SCET) threshold resummation to NNLL accuracy has been achieved in ref. [36] for direct photon production, in ref. [37] for the production of $W, Z$ bosons, and in ref. [38] for Higgs-boson production at high transverse momentum.

In this work, the threshold logarithms are resummed in Mellin moment space (see section 3 and section 4) as it is usually done in the direct QCD approach. It is also possible to resum in momentum space as it is usually done in the context of SCET. The two approaches to threshold resummation provide predictions of formally the same logarithmic accuracy and thus can be expected to deliver relatively similar resummed corrections. However, they obviously vary at the technical level, resulting in a different treatment of subleading corrections beyond the formal accuracy. The relation between the two approaches has been a subject of analytical studies [39-41] and numerical comparisons for various processes [40-44].

This work is organized as follows: in section 2, we briefly introduce the simplified dark-matter model. The kinematics and the partonic channels for dark-matter production in association with a jet are discussed in section 3. The resummation of the threshold logarithms is discussed in section 4 where we derive analytical results for the soft and the jet function and calculate the hard function numerically. In section 5 , numerical results for dark-matter production at the LHC are presented for several benchmark scenarios and the effects of resummation are investigated. Finally, we conclude in section 6 and present the analytic LO cross sections in appendix A.

\section{Dark-matter model}

The simplified dark-matter model which we investigate in this work consists of a massive s-channel vector mediator $Y$ and a Dirac dark-matter fermion $\chi$. The interaction part of the Lagrangian in the simplified dark matter model reads

$$
\mathcal{L}_{\text {int. }} \supset \bar{\chi} \gamma_{\mu}\left(g_{\chi}^{V}+g_{\chi}^{A} \gamma^{5}\right) \chi Y^{\mu}+\bar{q} \gamma_{\mu}\left(g_{\mathrm{SM}}^{V}+g_{\mathrm{SM}}^{A} \gamma^{5}\right) q Y^{\mu} .
$$

The first term in the Lagrangian describes the interaction of the mediator with the darkmatter fermions. We allow for a vector- and an axial-vector coupling. The second term describes the interaction of the mediator with the quark fields $q$. Again we allow for a vector- and an axial-vector coupling. In addition, we assume that the couplings are flavour diagonal so that no flavour mixing is introduced. Consequently, our simplified model has six free parameters, i.e.

$$
m_{Y}, m_{\chi}, g_{\chi}^{V}, g_{\chi}^{A}, g_{\mathrm{SM}}^{V}, g_{\mathrm{SM}}^{A} .
$$

The benchmark points considered in the numerical analysis are given by the fixed couplings

$$
g_{\chi}^{V}=1, g_{\chi}^{A}=0, g_{\mathrm{SM}}^{V}=0.25, g_{\mathrm{SM}}^{A}=0
$$

and we vary the masses of the mediator and the dark-matter particle. We stick to these values for the couplings which are often used as benchmark points in the literature although 
large regions of parameter space for the mediator and dark-matter masses are already excluded $[7,8]$. However, viable models can be obtained by choosing smaller couplings, e.g. $g_{\mathrm{SM}}^{V}=0.1$ or $g_{\mathrm{SM}}^{A}=0.1$. As discussed in section 5 , the exact choice for the couplings does not have a relevant impact on the relative size of the NLO or matched NLO+NLL' corrections.

\section{Partonic channels and kinematic considerations}

We calculate predictions for the production of a pair of dark-matter fermions $\chi$ recoiling against a jet $j$. At LO, the corresponding partonic processes are

$$
\begin{aligned}
& q \bar{q} \rightarrow \chi \bar{\chi}+g, \\
& q g \rightarrow \chi \bar{\chi}+q, \\
& \bar{q} g \rightarrow \chi \bar{\chi}+\bar{q},
\end{aligned}
$$

where $g$ denotes a gluon, $q$ a quark, and $\bar{q}$ an anti-quark. In section 4 , we label the different contributions to the NLL' cross section by the final-state parton at LO, e.g. the cross section $\mathrm{d} \sigma_{g}$ refers to the first process.

The central quantity for threshold resummation is the double differential cross section with respect to the transverse momentum $p_{T}$ of the pair of the dark-matter particles in the final state and their invariant mass $M^{2}$, i.e.

$$
\frac{\mathrm{d}^{2} \sigma(s)}{\mathrm{d} p_{T}^{2} \mathrm{~d} M^{2}}=\sum_{i, j} \int_{0}^{1} \mathrm{~d} x_{1} f_{i}\left(x_{1}\right) \int_{0}^{1} \mathrm{~d} x_{2} f_{j}\left(x_{2}\right) \frac{\mathrm{d}^{2} \hat{\sigma}_{i j}(\hat{s})}{\mathrm{d} p_{T}^{2} \mathrm{~d} M^{2}},
$$

where the squared partonic center-of-mass energy is given by $\hat{s}=x_{1} x_{2} s$ in terms of the squared collider energy $s$. The parton distribution functions (PDFs) are denoted by $f_{i}$ and the sums run over all contributing initial-state partons $i, j$.

The threshold energy $\sqrt{\hat{s}_{T}}$ to produce a final state with a given $p_{T}$ and $M^{2}$ is given by

$$
\sqrt{\hat{s}_{T}}=p_{T}+m_{T}
$$

with the transverse mass $m_{T}=\sqrt{p_{T}^{2}+M^{2}}$ and the corresponding partonic threshold variable is defined by

$$
\hat{y}_{T}=\frac{p_{T}+m_{T}}{\sqrt{\hat{s}}} .
$$

The LO cross section can be written as a function of the squared threshold variable $\hat{y}_{T}^{2}$ and the ratio $r=p_{T} / m_{T}$. The explicit results for the LO cross sections of the different partonic channels are given in appendix A. Since the dark-matter particles are not accessible experimentally, we have simplified the otherwise fully differential cross section by integrating over the dark-matter phase space. Hence, we obtain an effective $2 \rightarrow 2$ process which, for a given mass $M^{2}$, has the same kinematics as $2 \rightarrow 2$ SM processes for which threshold resummation has been obtained in the past $[23,37]$.

We perform resummation in Mellin space. The Mellin moments are defined as

$$
\frac{\mathrm{d}^{2} \hat{\sigma}_{i j}(N)}{\mathrm{d} p_{T}^{2} \mathrm{~d} M^{2}}=\int_{0}^{1} \mathrm{~d} \hat{y}_{T}^{2}\left(\hat{y}_{T}^{2}\right)^{N-1} \frac{\mathrm{d}^{2} \hat{\sigma}_{i j}\left(\hat{y}_{T}^{2}\right)}{\mathrm{d} p_{T}^{2} \mathrm{~d} M^{2}} .
$$


We do not require an analytic expression for the full NLO partonic cross section in this work. However, it is instructive to inspect its generic structure written in terms of a threshold expansion

$$
\frac{\mathrm{d}^{2} \hat{\sigma}_{i j}^{\mathrm{NLO}}(\hat{s})}{\mathrm{d} p_{T}^{2} \mathrm{~d} M^{2}}=\int_{0}^{1} \mathrm{~d} z \frac{\mathrm{d}^{2} \hat{\sigma}_{i j}^{\mathrm{LO}}(\hat{s} z)}{\mathrm{d} p_{T}^{2} \mathrm{~d} M^{2}}\left(c_{i j}^{(3)}\left[\frac{\log (1-z)}{1-z}\right]_{+}+c_{i j}^{(2)}\left[\frac{1}{1-z}\right]_{+}+c_{i j}^{(1)} \delta(1-z)\right)+d_{i j}\left(\hat{y}_{T}^{2}\right)
$$

with $z=1-s_{H} / Q^{2}$. Here, $s_{H}$ denotes the hadronic mass of the final state, and $Q^{2}=p_{T} \sqrt{\hat{s}_{T}}$ is a high-energy scale of this process which naturally appears in addition to $\hat{s}_{T}$. For initialstate parton combinations $i, j$ present at LO, the $z$-integration over the plus-distributions results in $\log ^{2}\left(1-\hat{y}_{T}^{2}\right)$ and $\log \left(1-\hat{y}_{T}^{2}\right)$ contributions at NLO. These threshold logarithms are resummed at NLL accuracy to all orders in this work. The function $d_{i j}$ denotes all terms which vanish in the threshold limit and are not needed at NLL' accuracy. The leading power coefficients of the threshold expansion $c_{i j}^{(3)}, c_{i j}^{(2)}$, and parts of $c_{i j}^{(1)}$ are derived analytically, while the so-called hard contributions to $c_{i j}^{(1)}$ are obtained numerically as discussed in section 4 . Note that

$$
Q^{2}=p_{T} \sqrt{\hat{s}_{T}}=\frac{p_{T}}{p_{T}+m_{T}} \hat{s}_{T}=\frac{r}{1+r} \hat{s}_{T} .
$$

This relation can be used in the soft and jet functions derived in section 4 to relate the two high-energy scales. As long as $r$ is of $\mathcal{O}(1)$, i.e. $p_{T}$ is of the same order as the mass of the dark-matter system, $\hat{s}_{T}$ and $Q^{2}$ are of the same order and standard threshold resummation holds without the need to take care of an additional large scale ratio.

As mentioned above, the distribution in $M^{2}$ is not accessible experimentally. The transverse momentum distribution or any fiducial cross section is simply obtained by integrating the resummed double-differential cross section, which is derived in section 4 .

\section{Resummed cross section}

In this section, we outline our strategy to calculate the resummed cross section at NLL' accuracy. For brevity, the double differential cross section introduced in section 3 is denoted by $\mathrm{d} \sigma$ in the following. We use the method of regions to derive all the one-loop ingredients of the resummation formula. The resummation is performed in Mellin space.

Using the method of regions, there are the soft, the collinear, and the hard region which contribute to the cross section near threshold at leading power. To define the different regions, we decompose the momentum $p$ of emitted radiation into components $\left(n_{+} \cdot p, n_{-} \cdot p, p_{\perp}\right)$, where we have introduced light-like four-vectors $n_{ \pm}$with spatial components along and opposite to the jet direction $\left(n_{ \pm}^{2}=0, n_{+} \cdot n_{-}=2\right)$. For the soft region $p$ scales according to $(\lambda, \lambda, \lambda)$, where the expansion parameter $\lambda=1-\hat{y}_{T}^{2}$ is small near threshold. The collinear and the hard regions scale according to $(1, \lambda, \sqrt{\lambda})$ and $(1,1,1)$, respectively.

To obtain the resummed cross section we have to calculate the leading-power result in each region. For the soft and the collinear region, our calculation leads to simple analytical results. The contribution of the hard region is obtained numerically as explained below. 
In the soft region, the leading-power expansion of the squared matrix elements for the real-emission contribution is identical to the Eikonal approximation for soft-gluon emission. Using the leading-power expansion also for the phase-space integration, one can write the soft contribution up to NLO for each partonic channel as a convolution with the LO cross section according to

$$
\mathrm{d} \hat{\sigma}_{a}^{\mathrm{NLO}, \text { soft }}=\int_{0}^{1} \mathrm{~d} z \mathrm{~d} \hat{\sigma}_{a}^{\mathrm{LO}}(z \hat{s}) S_{a}^{\mathrm{NLO}}(z),
$$

where the so-called soft function reads

$$
S_{a}^{\mathrm{NLO}}\left(z, \mu^{2}\right)=\delta(1-z)+C_{a} \frac{\alpha_{S}\left(\mu^{2}\right)}{\pi} \frac{(4 \pi)^{\epsilon}}{\Gamma(1-\epsilon)}\left(\frac{\mu^{2}}{\hat{s}_{T}}\right)^{\epsilon} \frac{1}{(1-z)^{1+2 \epsilon}}\left[-\frac{1}{\epsilon}+\mathcal{O}\left(\epsilon^{2}\right)\right] .
$$

The color factors are given by $C_{q}=C_{\bar{q}}=C_{F}+C_{A}-C_{F}=3$ and $C_{g}=2 C_{F}-C_{A}=-1 / 3$ for the partonic channels, i.e. each initial-state quark contributes $C_{F}$, an initial-state gluon $C_{A}$ and for each final-state particle the respective color factor is subtracted. Moreover, we work in $d=4-2 \varepsilon$ dimensions, and $\mu$ is the scale introduced in dimensional regularization to keep the running coupling dimensionless. In the calculation, we have used that the jet momentum is perpendicular to the beam direction at threshold. The term $(1-z)^{-(1+2 \epsilon)}$ can be expanded in $\epsilon$ in terms of a $\delta$-function and plus-distributions. In the following we consider the $\overline{\mathrm{MS}}$-renormalized quantity

$$
\begin{aligned}
S_{a}^{\mathrm{NLO}, R}\left(z, \mu^{2}\right)=\delta(1-z)+\frac{\alpha_{S}\left(\mu^{2}\right)}{\pi} C_{a} & {\left[\frac{1}{4}\left(\log ^{2} \frac{\mu^{2}}{\hat{s}_{T}}-\frac{\pi^{2}}{6}\right) \delta(1-z)\right.} \\
& \left.-\log \frac{\mu^{2}}{\hat{s}_{T}}\left[\frac{1}{1-z}\right]_{+}+2\left[\frac{\log (1-z)}{1-z}\right]_{+}\right] .
\end{aligned}
$$

At NLO, the soft function is, by construction, identical to the SCET soft function of [36], where a dimensionful convolution variable is used instead.

Since the Mellin transform converts convolutions like (4.1) into ordinary products, it is beneficial to go to Mellin space. The Mellin transform of the renormalized NLO soft function reads

$$
S_{a}^{\mathrm{NLO}}\left(N, \mu^{2}\right)=1+\frac{\alpha_{S}\left(\mu^{2}\right)}{\pi} C_{a}\left[\frac{1}{4} \log ^{2} \frac{\bar{N}^{2} \mu^{2}}{\hat{s}_{T}}+\frac{\pi^{2}}{8}+\mathcal{O}\left(\frac{1}{N}\right)\right],
$$

where we drop all terms suppressed by $1 / N$, since power-suppressed terms also contribute at $\mathcal{O}(1 / N)$, and use $\bar{N}=N \exp \left(\gamma_{E}\right)$ with Euler's constant $\gamma_{E}$. For the Mellin-space functions we drop the label $R$, since we always consider renormalized quantities. We observe that the characteristic scale of the soft function is given by $\mu_{s}^{2}=\hat{s}_{T} / \bar{N}^{2}$. The virtual contributions to the soft function consist of scaleless integrals and do not contribute.

In analogy, we can consider the collinear region which corresponds to real radiation collinear with the outgoing parton. The leading-power expansion of the squared matrixelement leads to the Born expression multiplied by the relevant splitting function for each partonic channel. Also expanding the phase space, in analogy to the soft case, one finds

$$
\mathrm{d} \hat{\sigma}_{a}^{\mathrm{NLO}, \text { coll }}=\int_{0}^{1} \mathrm{~d} z \mathrm{~d} \hat{\sigma}_{a}^{\mathrm{LO}}(z \hat{s}) J_{a}^{\mathrm{NLO}}(z)
$$


with the so-called jet function

$$
J_{a}^{\mathrm{NLO}}(z)=\delta(1-z)+\frac{\alpha_{S}\left(\mu^{2}\right)}{\pi} \frac{(4 \pi)^{\epsilon}}{\Gamma(1-\epsilon)}\left(\frac{\mu^{2}}{Q^{2}}\right)^{\epsilon} \frac{1}{(1-z)^{1+\epsilon}} j_{a}(\epsilon)
$$

for the outgoing parton $a$, where

$$
j_{q}(\epsilon)=\frac{C_{F}}{2}\left[-\frac{2}{\epsilon}-\frac{3}{2}+\left(\frac{\pi^{2}}{3}-\frac{7}{2}\right) \epsilon+\mathcal{O}\left(\epsilon^{2}\right)\right]
$$

for a quark or an anti-quark in the final state and

$$
j_{g}(\epsilon)=-\frac{C_{A}}{\epsilon}-\pi b_{0}+\frac{\epsilon}{18}\left(10 N_{f} T_{R}+\frac{C_{A}}{2}\left(6 \pi^{2}-67\right)\right)+\mathcal{O}\left(\epsilon^{2}\right)
$$

for a gluon, which can also split into $N_{f}=5$ light quark flavours. Here, $b_{0}=\frac{1}{12 \pi}\left(11 C_{A}-\right.$ $\left.4 T_{R} N_{f}\right)$ is the first coefficient of the QCD $\beta$-function and $T_{R}=1 / 2$ for QCD.

In analogy to the soft function, the renormalized NLO jet functions for outgoing partons in Mellin space read

$$
J_{q}^{\mathrm{NLO}}\left(N, \mu^{2}\right)=1+\frac{\alpha_{S}\left(\mu^{2}\right)}{\pi} C_{F}\left[\frac{1}{2} \log ^{2} \frac{\bar{N} \mu^{2}}{Q^{2}}+\frac{3}{4} \log \frac{\bar{N} \mu^{2}}{Q^{2}}+\frac{7}{4}-\frac{\pi^{2}}{6}+\mathcal{O}\left(\frac{1}{N}\right)\right]
$$

and

$$
\begin{aligned}
J_{g}^{\mathrm{NLO}}\left(N, \mu^{2}\right)=1+\frac{\alpha_{S}\left(\mu^{2}\right)}{\pi} & {\left[\frac{C_{A}}{2} \log ^{2} \frac{\bar{N} \mu^{2}}{Q^{2}}+\pi b_{0} \log \frac{\bar{N} \mu^{2}}{Q^{2}}\right.} \\
& \left.-\frac{1}{18}\left(10 N_{f} T_{R}+\frac{C_{A}}{2}\left(6 \pi^{2}-67\right)\right)+\mathcal{O}\left(\frac{1}{N}\right)\right] .
\end{aligned}
$$

We observe that the characteristic scale of the jet function is given by $\mu_{c}^{2}=Q^{2} / \bar{N}$. The virtual contributions to the jet functions again consist of scaleless integrals and do not contribute. The calculated jet functions agree with the SCET jet functions in ref. [36] at NLO.

Concerning the hard corrections, the real emission of a hard gluon is kinematically impossible near threshold. In contrast, the hard virtual contribution does not vanish. We do not attempt to derive or use analytical results for the hard contribution. Instead, we obtain this contribution numerically from the NLO MadGraph framework as detailed in the following. To access the virtual corrections at threshold, a MadLoop library for the process at hand is most suitable. To obtain the one-loop hard function for given $p_{T}$ and $M^{2}$, at leading power we only have to consider phase-space points at threshold. As for the LO cross section, we integrate the $\overline{\mathrm{MS}}$-renormalized virtual part of the NLO result over the dark-matter phase-space and divide the result by the respective LO result. Alternatively, one can use the process with a mediator of mass $M^{2}$ in the final state. At threshold, the phase-space is completely fixed up to an azimuthal symmetry and no integration is needed. One calculates the interference contribution of the $\overline{\mathrm{MS}}$-renormalized one-loop and the tree-level amplitude and normalizes by the corresponding squared tree-level amplitude.

The hard function for given $p_{T}$ and $M^{2}$ does neither depend on the dark-matter mass nor on the mediator mass. It depends only on the variables $Q^{2}$ and $\hat{s}_{T}$ representing the hard 
scale $\mu_{h}$, the scale $\mu$ and the renormalization scale $\mu_{R}$ since the renormalization of the strong coupling is part of the virtual corrections. Hence, the hard function includes logarithms of the scale ratio $\mu^{2} / \mu_{h}^{2}$. One can write the hard function as a scale independent piece given numerically and the scale-dependent terms which are available analytically. At the level of the complete matching coefficient, this is discussed at the end of the section, where we choose the hard scale $\mu_{h}^{2}=Q^{2}$ and the ratio $r=p_{T} / m_{T}$ as independent variables instead of $Q^{2}$ and $\hat{s}_{T}$. Since the hard function is a purely virtual correction, it is proportional to $\delta(1-z)$ and, therefore, it has no $N$-dependence in Mellin space.

In Mellin space, the threshold limit corresponds to large $N$. Hence, in the threshold limit, large logarithms can only be avoided in one of the three contributions (soft, collinear or hard) by a corresponding scale choice but not in all of them. The NLO result therefore necessarily contains large logarithms, no matter how the scale $\mu$ is chosen. Since the factorization scale, where the PDFs are evaluated, is usually taken as equal to the hard scale, we choose to use $\mu \sim \mu_{h}$. To control the large logarithms in the soft and the jet function, we use renormalization-group techniques as introduced in the following.

It can be shown that the cross section at leading power factorizes to all orders in perturbation theory $[24,36]$ and the Mellin-space cross section is given by an ordinary product of the LO cross section and the soft, the jet and the hard function. Using SCET convention [36] one writes

$$
\mathrm{d} \hat{\sigma}_{a}(N)=S_{a}(N+1) J_{a}(N+1) H_{a} \mathrm{~d} \hat{\sigma}_{a}^{\mathrm{LO}}(N) .
$$

Note that in this approach the physics of the incoming jets is completely contained in the soft function and only the hard collinear emissions of the outgoing jet give rise to the jet function. Hence, the assignment of the different physics contributions to the factorized functions differs compared to the direct QCD approach, cf. [23]. The factorized structure implies that the different pieces obey renormalization-group equations. For the soft and the jet function, one has

$$
\begin{aligned}
& \frac{\mathrm{d} \log S_{a}\left(N, \mu^{2}\right)}{\mathrm{d} \log \mu^{2}}=\gamma_{a}^{S_{A}}\left(\alpha_{S}\left(\mu^{2}\right)\right) \log \frac{\bar{N}^{2} \mu^{2}}{Q^{2}}-\gamma_{a}^{S_{B}}\left(\alpha_{S}\left(\mu^{2}\right)\right), \\
& \frac{\mathrm{d} \log J_{a}\left(N, \mu^{2}\right)}{\mathrm{d} \log \mu^{2}}=\gamma_{a}^{J_{A}}\left(\alpha_{S}\left(\mu^{2}\right)\right) \log \frac{\bar{N} \mu^{2}}{Q^{2}}-\gamma_{a}^{J_{B}}\left(\alpha_{S}\left(\mu^{2}\right)\right),
\end{aligned}
$$

where the anomalous dimensions $\gamma=\sum_{i}\left(\alpha_{S}\left(\mu^{2}\right) / \pi\right)^{i} \gamma^{(i)}$ are given as a power series in the strong coupling. The $\mathcal{O}\left(\alpha_{s}\right)$ coefficients can be directly read off the NLO expressions explicitly given before, i.e.

$$
\gamma_{a}^{S_{A}(1)}=\frac{C_{a}}{2}, \quad \gamma_{a}^{S_{B}(1)}=\frac{C_{a}}{2} \log \frac{1+r}{r}
$$

for the soft functions and

$$
\gamma_{q}^{J_{A}(1)}=C_{F}, \quad \gamma_{q}^{J_{B}(1)}=-\frac{3}{4} C_{F}, \quad \gamma_{g}^{J_{A}(1)}=C_{A}, \quad \gamma_{g}^{J_{B}(1)}=-\pi b_{0}
$$

for the jet functions. At NLL, the only necessary two-loop pieces are the $\mathcal{O}\left(\alpha_{s}^{2}\right)$ coefficients of $\gamma_{a}^{S_{A}}$ and $\gamma_{q, g}^{J_{A}}$. They are related to the process-independent cusp-anomalous dimension 
according to

$$
\gamma_{a}^{S_{A}}=\frac{C_{a}}{2} \gamma_{\text {cusp }}, \quad \gamma_{q}^{J_{A}}=C_{F} \gamma_{\text {cusp }}, \quad \gamma_{g}^{J_{A}}=C_{A} \gamma_{\text {cusp }}
$$

where

$$
\gamma_{\text {cusp }}^{(1)}=1 \quad \text { and } \quad \gamma_{\text {cusp }}^{(2)}=C_{A}\left(\frac{67}{36}-\frac{\pi^{2}}{12}\right)-\frac{5}{9} T_{R} N_{f}
$$

The soft function in Mellin space is perturbatively well-behaved for scales close to its natural scale, i.e. $\mu_{s}^{2} \sim \hat{s}_{T} / \bar{N}^{2}$, because no large logarithms appear. Given that $Q^{2} \sim \hat{s}_{T}$, we choose $Q^{2} / \bar{N}^{2}$ as the starting scale for the renormalization-group evolution. With this choice, our results for the exponentials derived below agree with ref. [23]. The solution of the renormalization-group equation reads

$$
\log \frac{S_{a}\left(N, \mu^{2}\right)}{S_{a}\left(N, Q^{2} / \bar{N}^{2}\right)}=-\int_{Q^{2} / \bar{N}^{2}}^{\mu^{2}} \frac{\mathrm{d} \hat{\mu}^{2}}{\hat{\mu}^{2}}\left(\int_{\mu^{2}}^{\hat{\mu}^{2}} \frac{\mathrm{d} q^{2}}{q^{2}} \gamma_{a}^{S_{A}}\left(\alpha_{S}\left(q^{2}\right)\right)+\gamma_{a}^{S_{B}}\left(\alpha_{S}\left(\hat{\mu}^{2}\right)\right)\right) .
$$

Separating and expanding the exponential containing only $\bar{N}$-independent terms to the desired order in $\alpha_{s}$, one can cast the resummed soft function into the form

$$
S_{a}\left(N, \mu^{2}\right)=\mathcal{S}_{a}\left(\mu^{2}\right) R^{\exp }\left(N, \mu^{2}, 2 \gamma_{a}^{S_{A}}, 2 \gamma_{a}^{S_{B}}, 2\right),
$$

where the exponential function for the resummed large logarithms reads

$$
\begin{aligned}
R^{\exp }\left(N, \mu^{2}, \gamma^{A}, \gamma^{B}, n\right)=\exp \left[-\int_{0}^{1-\frac{1}{N}} \frac{\mathrm{d} z}{1-z}(\right. & \int_{\mu^{2}}^{(1-z)^{n} Q^{2}} \frac{\mathrm{d} q^{2}}{q^{2}} \gamma^{A}\left(\alpha_{S}\left(q^{2}\right)\right) \\
& \left.\left.+\gamma^{B}\left(\alpha_{S}\left((1-z)^{n} Q^{2}\right)\right)\right)\right]
\end{aligned}
$$

and one has $\mathcal{S}_{a}\left(\mu^{2}\right)=S_{a}^{\mathrm{NLO}}\left(\mu^{2} / \bar{N}^{2}\right)$ at $\mathcal{O}\left(\alpha_{s}\right)$.

In analogy, the jet function in Mellin space is perturbatively well-behaved for $\mu_{c}^{2} \sim$ $Q^{2} / \bar{N}$. We choose $Q^{2} / \bar{N}$ as the starting scale for the renormalization-group evolution. The resummed jet function is then given by

$$
J_{a}\left(N, \mu^{2}\right)=\mathcal{J}_{a}\left(\mu^{2}\right) R^{\exp }\left(N, \mu^{2}, \gamma_{a}^{J_{A}}, \gamma_{a}^{J_{B}}, 1\right),
$$

where $\mathcal{J}_{a}\left(\mu^{2}\right)=J_{a}^{\mathrm{NLO}}\left(\mu^{2} / \bar{N}\right)$ at $\mathcal{O}\left(\alpha_{s}\right)$.

To summarize, the NLL' result reads

$$
\begin{aligned}
\mathrm{d} \hat{\sigma}_{a}^{\mathrm{NLL}^{\prime}}\left(N, \mu^{2}\right)=\mathrm{d} \hat{\sigma}_{a}^{\mathrm{LO}}(N) C_{a}\left(\mu^{2}\right) & R^{\exp }\left(N+1, \mu^{2}, 2 \gamma_{a}^{S_{A}}, 2 \gamma_{a}^{S_{B}}, 2\right) \\
& \times R^{\exp }\left(N+1, \mu^{2}, \gamma_{a}^{J_{A}}, \gamma_{a}^{J_{B}}, 1\right),
\end{aligned}
$$

where one needs $\gamma_{a}^{S_{A}}, \gamma_{a}^{J_{A}}$ up to $\mathcal{O}\left(\alpha_{s}^{2}\right)$ and $\gamma_{a}^{S_{B}}, \gamma_{a}^{J_{B}}$ up to $\mathcal{O}\left(\alpha_{s}\right)$. The matching coefficient

$$
C_{a}\left(\mu^{2}\right)=\mathcal{S}_{a}\left(\mu^{2}\right) \mathcal{J}_{a}\left(\mu^{2}\right) H_{a}\left(\mu^{2}\right)
$$

can be truncated at $\mathcal{O}\left(\alpha_{s}\right)$. The $\mathcal{O}\left(\alpha_{s}\right)$ contributions to $C_{a}\left(\mu^{2}\right)$ due to $\mathcal{S}_{a}\left(\mu^{2}\right)$ and $\mathcal{J}_{a}\left(\mu^{2}\right)$ are known analytically as discussed before, while the hard function is obtained numerically with the help of MadLoop. The Mellin transform of the leading order cross section 
$\mathrm{d}^{2} \hat{\sigma}_{a}^{\mathrm{LO}}(N) / \mathrm{d} p_{T}^{2} \mathrm{~d} M^{2}$ is calculated analytically and can be expressed in terms of hypergeometric functions, see appendix A.

The resummed exponentials in Mellin space agree with the results in ref. [23], where one can also find analytic expressions for the integrals in the exponential. As in ref. [23], for our numerical results, we resum large logarithms of $N$ instead of $\bar{N}$, i.e. $N$-independent exponentials containing $\gamma_{E}$ are expanded to $\mathcal{O}\left(\alpha_{s}\right)$ and lead to additional terms proportional to $\gamma_{E}$ and $\gamma_{E}^{2}$ in $\mathcal{S}_{a}\left(\mu^{2}\right)$ and $\mathcal{J}_{a}\left(\mu^{2}\right)$.

The scale $\mu$ is physically relevant because we define the PDFs at $\mu_{F}$. Hence, we have to identify $\mu=\mu_{F}$. We have checked that the $\mu_{F}$-dependence of the matching coefficient is given by the known logarithms introduced via the PDF renormalization. In addition, we have to define the numerical input for the strong coupling constant at some scale $\mu_{R}$. Within the matching coefficient, all terms of $\mathcal{O}\left(\alpha_{s}\right)$ are simply evaluated using $\alpha_{S}\left(\mu_{R}^{2}\right)$. Hence the matching coefficient depends also on the choice of $\mu_{R}$. The renormalization of the strong coupling in the LO cross section is part of the hard function. Also $R^{\exp }$ depends on the input value $\alpha_{S}\left(\mu_{R}^{2}\right)$ from which $\alpha_{S}$ at another scale is derived using its renormalizationgroup running (see e.g. ref. [23]). The matching coefficient can be written as

$$
C_{a}\left(\mu_{F}^{2}, \mu_{R}^{2}\right)=\left(1+\frac{\alpha_{S}\left(\mu_{R}^{2}\right)}{\pi}\left(\hat{C}_{a}+\left(\mathcal{P}_{a}-2 \gamma_{E} \gamma_{a}^{S_{A}(1)}-\gamma_{E} \gamma_{a}^{J_{A}(1)}\right) \log \frac{Q^{2}}{\mu_{F}^{2}}-\pi b_{0} \log \frac{Q^{2}}{\mu_{R}^{2}}\right)\right)
$$

where $\mathcal{P}_{g}=\frac{3}{2} C_{F}$ and $\mathcal{P}_{q}=\mathcal{P}_{\bar{q}}=\frac{3}{4} C_{F}+\pi b_{0}$. The constants $\mathcal{P}_{a}$ are related to PDF renormalization, where the $C_{F}$-pieces originate from the renormalization of the quark PDF and the piece proportional to $b_{0}$ from the renormalization of the gluon PDF. The terms proportional to $\gamma_{E}$ arise because we only keep $\log N$ instead of $\log \bar{N}$ in the exponentials, as mentioned above. The functions $\hat{C}_{a}$ contain all scale-independent terms and include the hard contributions which are only available numerically. The scale independent parts $\hat{C}_{a}$ of the matching coefficients only depend on the ratio $r=p_{T} / m_{T}$ and are presented in figure 1 . The tabulated data of figure 1 is available as supplementary material of this publication.

To obtain numerical results from the resummed expression, we have to perform the inverse Mellin transform of the Mellin-space cross section, i.e.

$$
\mathrm{d} \sigma_{a}^{\mathrm{NLL}^{\prime}}(s)=\frac{1}{2 \pi i} \int_{c-i \infty}^{c+i \infty} \mathrm{d} N\left(y_{T}^{2}\right)^{-N} \sum_{i, j} f_{i}(N+1) f_{j}(N+1) \mathrm{d} \hat{\sigma}_{a}^{\mathrm{NLL}^{\prime}}(N),
$$

where $y_{T}=\left(p_{T}+m_{T}\right) / \sqrt{s}$ is the hadronic threshold variable and $f_{i}(N)$ are the Mellin moments of the PDFs. Following the minimal prescription [45], the constant $c$ is chosen to the left of the poles due to the running of the strong coupling $\alpha_{s}$ in the exponents of $\mathrm{d} \hat{\sigma}_{a}^{\mathrm{NLL}}(N)$, but to the right of all other poles of the integrand. To use the standard PDF implementations, which are not available in Mellin space, we rewrite the resummed hadronic cross section according to [46]

$$
\mathrm{d} \sigma_{a}^{\mathrm{NLL}^{\prime}}(s)=\sum_{i, j} \int_{0}^{1} \mathrm{~d} x_{1} \tilde{f}_{i}\left(x_{1}\right) \int_{0}^{1} \mathrm{~d} x_{2} \tilde{f}_{j}\left(x_{2}\right) \mathrm{d} \tilde{\sigma}_{a}^{\mathrm{NLL}^{\prime}}(\hat{s}) .
$$

Here, $\mathrm{d} \tilde{\sigma}_{a}^{\mathrm{NLL}^{\prime}}(\hat{s})$ is the inverse Mellin transform of $\mathrm{d} \hat{\sigma}_{a}^{\mathrm{NLL}^{\prime}}(N) / N^{2}$ with respect to the partonic threshold variable. Moreover, the $\tilde{f}_{i}$ are the inverse Mellin transforms of 


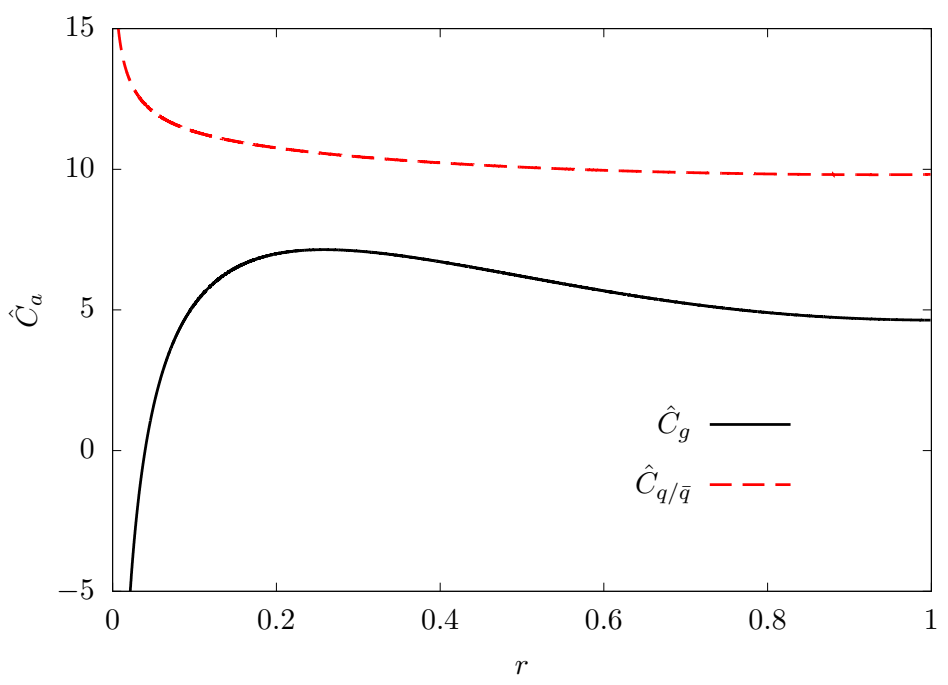

Figure 1. The scale independent part $\hat{C}_{a}$ of the matching coefficient as a function of $r=p_{T} / m_{T}$ for the two partonic channels as defined in (4.23).

$N f_{i}(N+1)$, which are given by derivatives of the standard PDF functions according to $\tilde{f}_{i}(x)=\frac{\mathrm{d}}{\mathrm{d} x}\left(-x f_{i}(x)\right)$, and the sums run over all initial-state partons $i, j$ contributing to the LO process with parton $a$ in the final state. Introducing the convergence factor $1 / N^{2}$ before performing the inverse Mellin transform of the resummed partonic cross section makes the numerical integration feasible.

\section{$5 \quad$ Numerical results}

In this section, we discuss numerical results for benchmark scenarios within the simplified model discussed in section 2. We compute the full NLO predictions using MadGraph5_aMC@NLO [18] using a FeynRules [19, 20] implementation of the investigated dark-matter model which is publicly available [13, 47]. The NLL' prediction which has been discussed in detail in section 4 is matched to this NLO result. To avoid double counting, we add the NLO and the NLL' prediction and subtract the expansion of the resummed result to next-to-leading order in the strong coupling $\alpha_{s}$. Hence, only the contributions of the resummed cross section beyond NLO are taken into account.

We use the PDF set PDF4LHC15_nlo_100 [48-51] as provided by LHAPDF [52] throughout our analysis, i.e. for LO, NLO and NLO+NLL' predictions. Our central scale choice is given by

$$
\mu_{F}=\mu_{R}=\frac{H_{T}}{2}=\frac{m_{T}}{2}+\sum_{j} \frac{p_{T}^{j}}{2},
$$

where the sum runs over all final state jets. This is a common scale choice in fixedorder calculations for processes involving jet production, cf. [13]. Moreover, $H_{T} / 2$ is a scale choice close to the two hard threshold variables $\sqrt{\hat{s}_{T}}$ and $Q$, since at threshold $\sqrt{\hat{s}_{T}}=m_{T}+p_{T}=H_{T}$ and $Q^{2}=\frac{r}{1+r} H_{T}^{2} \leq H_{T}^{2} / 2$. We use $\alpha_{S}\left(\mu_{R}^{2}\right)$ as provided by LHAPDF 
for the given PDF set. Moreover, we use $N_{f}=5$ as the number of light flavours. We present predictions for the LHC at the center-of-mass energy $\sqrt{s}=13 \mathrm{TeV}$. We use a fixed width for the mediator which is calculated at $\mathrm{LO}$ as discussed in appendix $\mathrm{A}$.

The theoretical uncertainty of the NLO and the matched NLO+NLL' results due to missing higher-order corrections is estimated in the usual way by independently varying the factorization scale $\mu_{F}$ and the renormalization scale $\mu_{R}$. To be precise, we use a standard 7 -point variation, i.e. we make predictions for

$$
\mu_{F / R}=\xi_{F / R} \frac{H_{T}}{2},\left(\xi_{F}, \xi_{R}\right)=(1,1),(0.5,0.5),(2,2),(1,0.5),(1,2),(0.5,1),(2,1)
$$

and show the minimal and maximal prediction as an error band around the nominal prediction $\left(\xi_{F}, \xi_{R}\right)=(1,1)$.

As discussed in section 2, the vector and axial-vector couplings of the mediator to the dark-matter particles and the standard-model quarks are set to

$$
g_{\chi}^{V}=1, g_{\chi}^{A}=0, g_{\mathrm{SM}}^{V}=0.25, g_{\mathrm{SM}}^{A}=0,
$$

respectively. As can be seen in the analytic LO results presented in appendix A, the couplings to the SM quarks $g_{\mathrm{SM}}^{V, A}$ enter the cross section explicitly as the sum of the squared vector and axial-vector couplings and in the mediator width. Hence, changing $g_{\mathrm{SM}}^{V, A}$ mainly rescales the cross section by an overall factor which depends on whether the mediator is produced on-shell or off-shell. To a good approximation, the cross section also only depends on the squared sum of $g_{\chi}^{V, A}$ if the mediator mass is much larger than the dark-matter mass and the production is dominated by mediators close to their mass shell. For $m_{Y}<m_{\chi}$, the production involves off-shell mediators and it is velocity suppressed near $M^{2}=4 m_{\chi}^{2}$ for axial-vector couplings. Hence, for axial-vector couplings the differential cross-section with respect to $M^{2}$ peaks at higher $M^{2}$, the cross section will be smaller compared to a model with a vector coupling of the same size but no qualitative differences are expected for the radiative corrections.

The observable of interest is the missing transverse-momentum distribution. To obtain the $p_{T, \text { miss }}$ spectrum, the double-differential resummed result discussed in section 4 is numerically integrated with respect to the invariant mass $M^{2}$ of the dark-matter system.

At NLO precision, the model has been already investigated in ref. [13]. Using the setup of [13], we have reproduced the NLO cross section at the level of $0.5 \%$ or better. For the LO results and the corresponding K-factors we have observed substantial differences. It turned out that the LO cross sections in ref. [13] have not been obtained with the PDF set stated in the paper.

We discuss three benchmark points in detail. We have chosen two parameter sets for the mediator mass $m_{Y}$ and the dark-matter mass $m_{\chi}$ which lead to a large invariant mass of the dark-matter pair. In this case, the resummed cross section can be expected to reliably estimate the higher-order corrections beyond NLO accuracy. The first benchmark point is $\left(m_{Y}, m_{\chi}\right)=(100,500) \mathrm{GeV}$, i.e. a relatively light mediator with a heavier darkmatter particle leading to off-shell production. The second benchmark point is $\left(m_{Y}, m_{\chi}\right)=$ $(1000,50) \mathrm{GeV}$, i.e. a heavy mediator with a relatively light dark-matter particle, which 


\begin{tabular}{|c|l|l|l|}
\hline $\begin{array}{c}\left(m_{Y}, m_{\chi}\right) \\
{[\mathrm{GeV}]}\end{array}$ & & $p_{T, \text { miss }}>500 \mathrm{GeV}$ & $p_{T, \text { miss }}>1000 \mathrm{GeV}$ \\
\hline \multirow{5}{*}{$(100,500)$} & $\sigma_{\mathrm{LO}}[\mathrm{pb}]$ & $5.18_{-0.86}^{+1.11} \times 10^{-4}$ & $3.85_{-0.71}^{+0.94} \times 10^{-5}$ \\
& $\sigma_{\mathrm{NLO}}[\mathrm{pb}]$ & $6.96_{-0.51}^{+0.43} \times 10^{-4}$ & $5.15_{-0.41}^{+0.32} \times 10^{-5}$ \\
& $\sigma_{\mathrm{NLO}+\mathrm{NLL}},[\mathrm{pb}]$ & $7.25_{-0.24}^{+0.22} \times 10^{-4}$ & $5.41_{-0.16}^{+0.14} \times 10^{-5}$ \\
& $\mathrm{~K}_{\mathrm{NLO} / \mathrm{LO}}$ & 1.34 & 1.34 \\
& $\mathrm{~K}_{\mathrm{NLO}+\mathrm{NLL}} / \mathrm{LO}$ & 1.40 & 1.40 \\
\hline \multirow{5}{*}{$(1000,50)$} & $\sigma_{\mathrm{LO}}[\mathrm{pb}]$ & $6.65_{-1.07}^{+1.37} \times 10^{-2}$ & $3.85_{-0.70}^{+0.92} \times 10^{-3}$ \\
& $\sigma_{\mathrm{NLO}}[\mathrm{pb}]$ & $8.74_{-0.60}^{+0.48} \times 10^{-2}$ & $5.11_{-0.40}^{+0.30} \times 10^{-3}$ \\
& $\sigma_{\mathrm{NLO}+\mathrm{NLL}},[\mathrm{pb}]$ & $9.03_{-0.29}^{+0.26} \times 10^{-2}$ & $5.34_{-0.16}^{+0.13} \times 10^{-3}$ \\
& $\mathrm{~K}_{\mathrm{NLO} / \mathrm{LO}}$ & 1.31 & 1.33 \\
& $\mathrm{~K}_{\mathrm{NLO}+\mathrm{NLL} / \mathrm{LO}}$ & 1.36 & 1.39 \\
\hline \multirow{5}{*}{$(100,1)$} & $\sigma_{\mathrm{LO}}[\mathrm{pb}]$ & $7.26_{-1.08}^{+1.36} \times 10^{-1}$ & $1.45_{-0.25}^{+0.33} \times 10^{-2}$ \\
& $\sigma_{\mathrm{NLO}}[\mathrm{pb}]$ & $1.08_{-0.09}^{+0.09} \times 10^{0}$ & $2.15_{-0.20}^{+0.19} \times 10^{-2}$ \\
& $\sigma_{\mathrm{NLO}+\mathrm{NLL}},[\mathrm{pb}]$ & $1.10_{-0.05}^{+0.06} \times 10^{0}$ & $2.22_{-0.09}^{+0.10} \times 10^{-2}$ \\
& $\mathrm{~K}_{\mathrm{NLO} / \mathrm{LO}}$ & 1.48 & 1.48 \\
& $\mathrm{~K}_{\mathrm{NLO}+\mathrm{NLL} / \mathrm{LO}}$ & 1.51 & 1.53 \\
\hline
\end{tabular}

Table 1. Integrated cross section for a given cut on $p_{T, \text { miss }}$ for our benchmark points at different levels of theoretical accuracy and the corresponding K-factors. The stated errors are determined by the 7-point scale variation defined in (5.1).

can be produced from an on-shell mediator. For these parameter points the resummed prediction expanded to NLO is slightly larger than the full NLO result and reproduces it within $5 \%$ throughout the considered range for $p_{T, \text { miss }}$. In addition, we investigate the model with the mass combination $\left(m_{Y}, m_{\chi}\right)=(100,1) \mathrm{GeV}$, i.e. a relatively light mediator with almost massless dark-matter particles. This mass combination is kinematically very close to the main SM-background process, i.e. Z-boson production in association with a high- $p_{T}$ jet with subsequent invisible Z-boson decays into neutrinos. Since the final-state dark-matter system is rather light in this case, the difference between the NLO-expanded resummed result and the full NLO prediction is approximately $-10 \%$ of the NLO result at intermediate $p_{T, \text { miss }}$ values around $500 \mathrm{GeV}$ and is reduced to approximately $-5 \%$ at $p_{T, \text { miss }}=2 \mathrm{TeV}$.

For concreteness, we present integrated cross sections with a given cut on $p_{T \text {,miss }}$ for our benchmark points in table 1 . The LO, NLO, and matched NLO+NLL' results are shown along with the corresponding K-factors.

The $p_{T \text {,miss }}$ distributions are shown in figure 2 and figure 3 in the range between $150 \mathrm{GeV}$ and $2 \mathrm{TeV}$. The NLO K-factor for the heavy dark-matter systems in figure 2 does not vary much as a function of $p_{T, \text { miss }}$ and amounts to $30-40 \%$. For the lighter dark-matter 

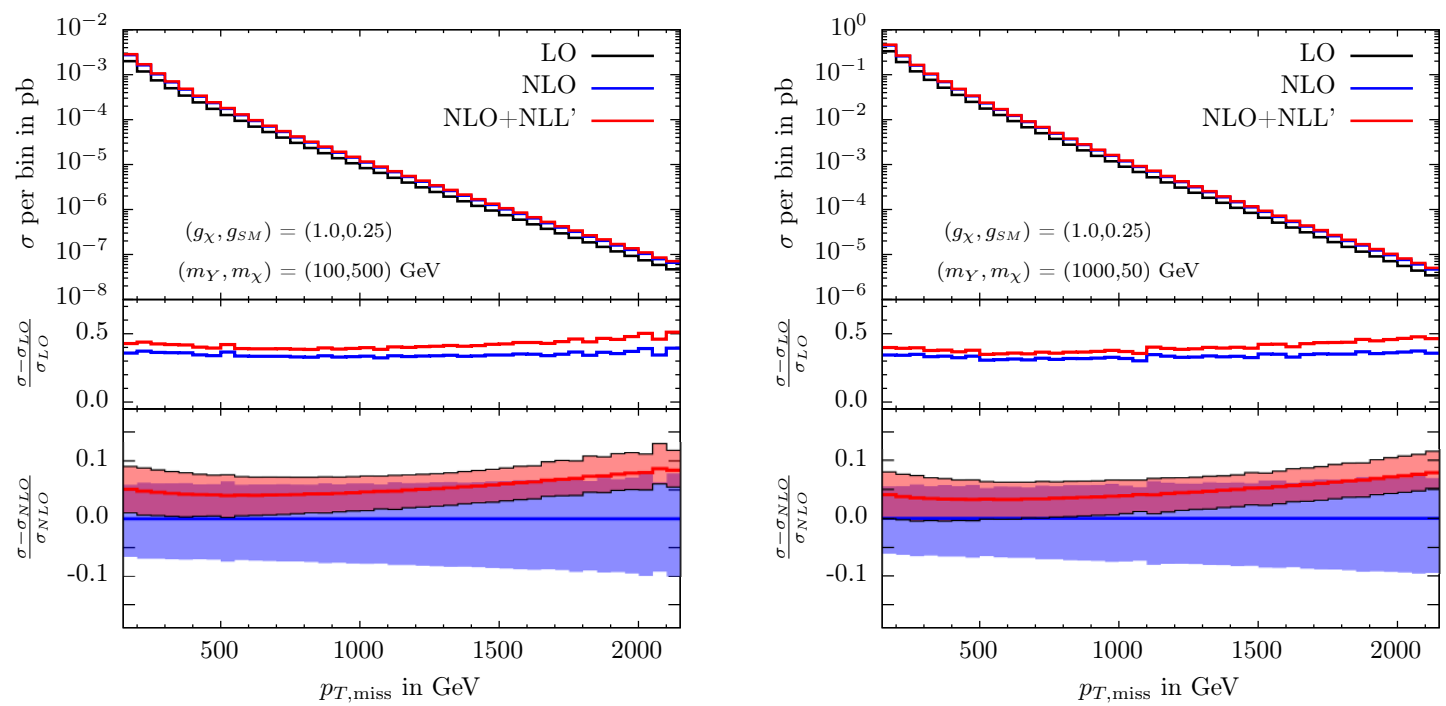

Figure 2. The missing transverse-momentum distribution for $\left(m_{Y}, m_{\chi}\right)=(100,500) \mathrm{GeV}$ (left) and $\left(m_{Y}, m_{\chi}\right)=(1000,50) \mathrm{GeV}$ (right) is shown in the upper panel at LO, NLO, and matched $\mathrm{NLO}+\mathrm{NLL}$ ' accuracy. In addition, the K-factors with respect to the LO prediction (middle panel) and the scale variation of the NLO and matched NLO+NLL' predictions (lower panel) are displayed.

system in figure 3 the NLO K-factor varies from 40 to 50\%. For mediator and dark-matter masses below $100 \mathrm{GeV}$, the K-factor grows and the accuracy of the expanded NLL' result is getting worse. For example, one finds $\mathrm{K} \sim 1.7$ for $\left(m_{Y}, m_{\chi}\right)=(10,1) \mathrm{GeV}$ for a $p_{T, \text { miss }}$ cut of $500 \mathrm{GeV}$. For smaller masses, di-jet final-states start to sizeably contribute, where the mediator is emitted from one of the jets. These contributions cannot be expected to be well approximated by the threshold behavior of the mono-jet final-state. Hence, in the following, we do not consider final states, for which the invariant mass of the dark-matter system is small.

For the benchmark points with a massive dark-matter final state shown in figure 2, the resummation adds roughly $5 \%$ of the LO predictions on top of the NLO prediction in the region where the LHC is most sensitive. In the very tails of the distributions, the corrections due to resummation increases up to $12 \%$. Hence, as expected, the largest impact is observed in the tail of the $p_{T, \text { miss }}$-distribution at $2 \mathrm{TeV}$, where the dark-matter production takes place closer to threshold. For $\left(m_{Y}, m_{\chi}\right)=(100,1) \mathrm{GeV}$, the correction is almost negligible at small $p_{T \text {,miss }}$ but also rises to almost $10 \%$ of the LO result in the tail of the distribution at $2 \mathrm{TeV}$. Note that it is essential to take into account the $\mathcal{O}\left(\alpha_{s}\right)$ terms of the matching coefficient in (4.23) on top of the resummed exponentials. Using NLL instead of NLL' results, the full NLO result is not approximated well by the expanded resummed result and the correction beyond NLO is much smaller.

The matched NLO+NLL' prediction also considerably reduces the scale uncertainty at large transverse momenta. At large $p_{T \text {,miss }}$ above $1 \mathrm{TeV}$ the NLO error band is reduced by roughly $50 \%$ for all three benchmark scenarios. For smaller values of $p_{T, \text { miss }}$ the reduction of the size of the error band decreases with decreasing $p_{T \text {,miss }}$, as shown in figure 3 . As 


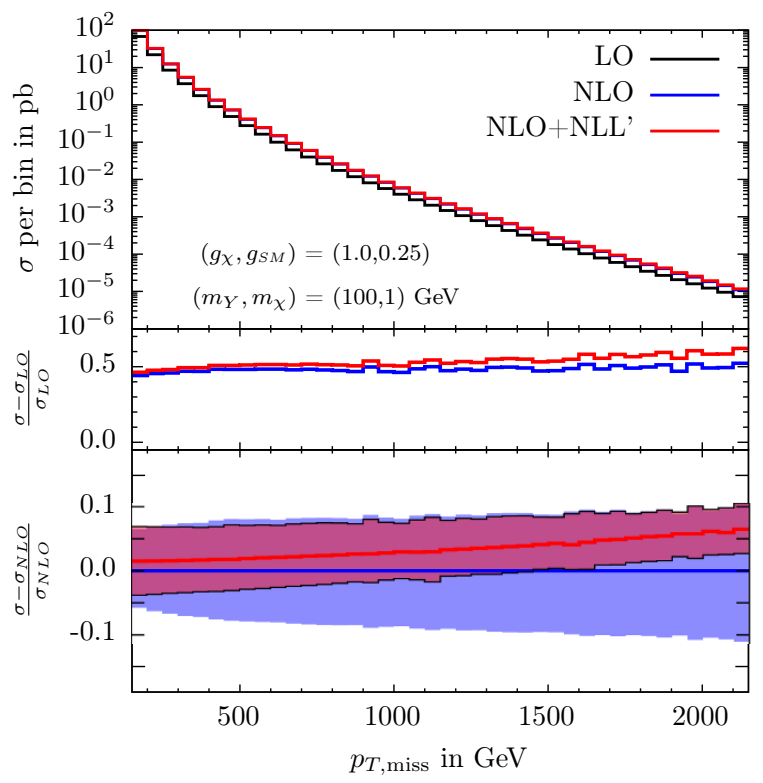

Figure 3. The missing transverse-momentum distribution for $\left(m_{Y}, m_{\chi}\right)=(100,1) \mathrm{GeV}$ is shown in the upper panel at $\mathrm{LO}, \mathrm{NLO}$, and matched NLO+NLL' accuracy. In addition, the K-factors with respect to the LO prediction (middle panel) and the scale variation of the NLO and matched $\mathrm{NLO}+\mathrm{NLL}$ ' predictions (lower panel) are displayed.

expected, these results reproduce known results for vector-boson plus jet production in the Standard Model [37]. Note that the reduction of the scale error is less pronounced if we define the scale-variation error by a nine-point variation, i.e. also including $\left(\xi_{F}, \xi_{R}\right)=$ $(0.5,2),(2,0.5)$. The NLO error band is not affected by the different definition, while the width of the matched NLO+NLL' scale-variation band is increased at the upper end. Using the nine-point variation, there is no relevant improvement in the scale uncertainty for small transverse momenta. For $\left(m_{Y}, m_{\chi}\right)=(100,1) \mathrm{GeV}$, the width of the matched NLO+NLL' error band is even bigger than the NLO error band. However, the error band of the fixedorder prediction grows to more than $15 \%$ at transverse momenta close to $2 \mathrm{TeV}$, while the width of the error band for the matched predictions stays more or less constant at $10 \%$, using the nine-point scale variation.

\section{Conclusions}

We have computed the resummed cross section for the hadroproduction of a dark-matter fermion pair in a simplified dark-matter model to NLO+NLL' accuracy. We have derived the corresponding soft and jet functions analytically using the strategy of regions. The hard function has been computed numerically using MadLoop. The resummation has been performed in Mellin-space, and we have matched the resummed results to the full fixedorder NLO predictions obtained with MadGraph5_aMC@NLO.

We have presented numerical results for dark-matter production at the LHC for various mediator and dark-matter masses. For dark-matter pairs produced with a large invariant 
mass in the TeV range, resummation increases the NLO prediction by approximately $5 \%$ to $10 \%$. For smaller invariant masses of the dark-matter pair down to $100 \mathrm{GeV}$, the increase of the cross section beyond the NLO prediction is slightly reduced. For even smaller invariant masses, the threshold resummation as a means to estimate the leading higher-order corrections becomes questionable. Note that it is essential to include the NLO matching coefficient within the resummed result. We also observe a significant reduction in the scale uncertainty of the predictions, where the size of the NLO error band is reduced by $50 \%$ at large transverse momentum.

The combination of simple analytical formulas with numerical results from the MadGraph framework allows one to obtain the NLO+NLL' predictions for dark-matter production at the LHC in a straightforward manner.

\section{Acknowledgments}

This work has been supported by the German Research Foundation (DFG) through the Research Unit "New Physics at the Large Hadron Collider" (FOR 2239) and the CRC/Transregio "P3H: Particle Physics Phenomenology after the Higgs Discovery" (TRR 257). We are grateful to Kentarou Matawari for discussions and the comparison of numerical results.

\section{A LO cross sections}

To formulate the resummation in Mellin space, we need the Mellin moments of the leadingorder differential cross section for the two partonic channels. The double-differential cross sections are given by

$$
\frac{\mathrm{d}^{2} \hat{\sigma}_{a}^{\mathrm{LO}}}{\mathrm{d} p_{T}^{2} \mathrm{~d} M^{2}}=\frac{2 \alpha_{s}}{\pi} \frac{\left(g_{\mathrm{SM}}^{V}\right)^{2}+\left(g_{\mathrm{SM}}^{A}\right)^{2}}{m_{T}^{2} p_{T}^{2}} \frac{\hat{y}_{T}^{2}}{\sqrt{1-\hat{y}_{T}^{2}}} \frac{M \Gamma_{Y}^{\chi}\left(M, m_{\chi}\right)}{\left(M^{2}-m_{Y}^{2}\right)^{2}+\Gamma_{Y}^{2} m_{Y}^{2}} f_{a}\left(\hat{y}_{T}^{2}, r\right),
$$

where

$$
\Gamma_{Y}^{\chi}(M, m)=\frac{M}{12 \pi} \sqrt{1-\frac{4 m^{2}}{M^{2}}}\left(\left(g_{\chi}^{V}\right)^{2}\left(1+\frac{2 m^{2}}{M^{2}}\right)+\left(g_{\chi}^{A}\right)^{2}\left(1-\frac{4 m^{2}}{M^{2}}\right)\right)
$$

results from the inclusive decay of the mediator and

$$
\begin{aligned}
f_{g}\left(\hat{y}_{T}^{2}, r\right) & =\frac{2(r-1)^{2} \hat{y}_{T}^{4}-4 r^{2} \hat{y}_{T}^{2}+2(r+1)^{2}}{9(r+1)^{3} \sqrt{(r+1)^{2}-\hat{y}_{T}^{2}(r-1)^{2}}}, \\
f_{q}\left(\hat{y}_{T}^{2}, r\right) & =\frac{2(r-1)^{3} \hat{y}_{T}^{6}+(r-2)(r-1)(r+2) \hat{y}_{T}^{4}+(r+1)\left(4 r^{2}-3\right) \hat{y}_{T}^{2}+(r+1)^{3}}{24(r+1)^{4} \sqrt{(r+1)^{2}-\hat{y}_{T}^{2}(r-1)^{2}}}
\end{aligned}
$$

are functions which are specific for the given partonic production process. Moreover, we use a fixed width in the propagator of the mediator given by

$\Gamma_{Y}=\Gamma_{Y}^{\chi}\left(m_{Y}, m_{\chi}\right) \Theta\left(m_{Y}^{2}-4 m_{\chi}^{2}\right)+N_{C} \Gamma_{Y}^{\mathrm{SM}}\left(m_{Y}, m_{t}\right) \Theta\left(m_{Y}^{2}-4 m_{t}^{2}\right)+N_{f} N_{C} \Gamma_{Y}^{\mathrm{SM}}\left(m_{Y}, 0\right)$, 
where the theta-functions determine which decay channels are kinematically accessible for a given mediator mass, and $\Gamma_{Y}^{S M}$ is obtained from $\Gamma_{Y}^{\chi}$ by replacing the couplings to the dark-matter particle by the SM couplings to the quarks. To be specific, we use $m_{t}=172 \mathrm{GeV}$, and the width of the mediator for the benchmark points is given by $\Gamma_{Y}=$ $(0.056,0.025,0.051) m_{Y}$ for $\left(m_{Y}, m_{\chi}\right)=(1000,50),(100,500),(100,1) \mathrm{GeV}$, respectively.

For the resummed cross section, we need the Mellin-space representation of the cross section according to

$$
\frac{\mathrm{d}^{2} \hat{\sigma}_{a}^{\mathrm{LO}}(N)}{\mathrm{d} p_{T}^{2} \mathrm{~d} M^{2}}=\int_{0}^{1} \mathrm{~d} \hat{y}_{T}^{2}\left(\hat{y}_{T}^{2}\right)^{N-1} \frac{\mathrm{d}^{2} \hat{\sigma}_{a}^{\mathrm{LO}}\left(\hat{y}_{T}^{2}\right)}{\mathrm{d} p_{T}^{2} \mathrm{~d} M^{2}}
$$

Using

$$
\int_{0}^{1} \mathrm{~d} \hat{y}_{T}^{2}\left(\hat{y}_{T}^{2}\right)^{N-1} \frac{\left(\hat{y}_{T}^{2}\right)^{n}}{\sqrt{1-\hat{y}_{T}^{2}} \sqrt{1-z \hat{y}_{T}^{2}}}=\frac{\sqrt{\pi} \Gamma(N+n){ }_{2} F_{1}\left(\frac{1}{2}, N+n ; N+\frac{2 n+1}{2} ; z\right)}{\Gamma\left(N+\frac{2 n+1}{2}\right)},
$$

it can be expressed in terms of hypergeometric functions, where $z=\frac{(r-1)^{2}}{(r+1)^{2}}$.

Open Access. This article is distributed under the terms of the Creative Commons Attribution License (CC-BY 4.0), which permits any use, distribution and reproduction in any medium, provided the original author(s) and source are credited.

\section{References}

[1] Particle Data Group collaboration, Review of Particle Physics, Phys. Rev. D 98 (2018) 030001 [INSPIRE].

[2] F. Kahlhoefer, Review of LHC Dark Matter Searches, Int. J. Mod. Phys. A 32 (2017) 1730006 [arXiv: 1702 .02430] [INSPIRE].

[3] J.M. Lindert et al., Precise predictions for $V+$ jets dark matter backgrounds, Eur. Phys. J. C 77 (2017) 829 [arXiv:1705.04664] [INSPIRE].

[4] O. Buchmueller, M.J. Dolan and C. McCabe, Beyond Effective Field Theory for Dark Matter Searches at the LHC, JHEP 01 (2014) 025 [arXiv: 1308.6799] [INSPIRE].

[5] J. Abdallah et al., Simplified Models for Dark Matter and Missing Energy Searches at the $L H C$, arXiv: 1409.2893 [INSPIRE].

[6] J. Abdallah et al., Simplified Models for Dark Matter Searches at the LHC, Phys. Dark Univ. 9-10 (2015) 8 [arXiv:1506.03116] [INSPIRE].

[7] CMS collaboration, Search for narrow and broad dijet resonances in proton-proton collisions at $\sqrt{s}=13 \mathrm{TeV}$ and constraints on dark matter mediators and other new particles, JHEP 08 (2018) 130 [arXiv: 1806.00843] [INSPIRE].

[8] ATLAS collaboration, Constraints on mediator-based dark matter models using $\sqrt{s}=13 \mathrm{TeV}$ pp collisions at the LHC with the ATLAS detector, ATLAS-CONF-2018-051.

[9] M. Fairbairn, J. Heal, F. Kahlhoefer and P. Tunney, Constraints on $Z^{\prime}$ models from LHC dijet searches and implications for dark matter, JHEP 09 (2016) 018 [arXiv: 1605.07940] [INSPIRE]. 
[10] P.J. Fox and C. Williams, Next-to-Leading Order Predictions for Dark Matter Production at Hadron Colliders, Phys. Rev. D 87 (2013) 054030 [arXiv:1211.6390] [InSPIRE].

[11] U. Haisch, F. Kahlhoefer and E. Re, QCD effects in mono-jet searches for dark matter, JHEP 12 (2013) 007 [arXiv:1310.4491] [INSPIRE].

[12] D. Abercrombie et al., Dark Matter Benchmark Models for Early LHC Run-2 Searches: Report of the ATLAS/CMS Dark Matter Forum, arXiv:1507.00966 [INSPIRE].

[13] M. Backović, M. Krämer, F. Maltoni, A. Martini, K. Mawatari and M. Pellen, Higher-order QCD predictions for dark matter production at the LHC in simplified models with s-channel mediators, Eur. Phys. J. C 75 (2015) 482 [arXiv: 1508.05327] [INSPIRE].

[14] M. Neubert, J. Wang and C. Zhang, Higher-Order QCD Predictions for Dark Matter Production in Mono-Z Searches at the LHC, JHEP 02 (2016) 082 [arXiv:1509.05785] [INSPIRE].

[15] G. Das, C. Degrande, V. Hirschi, F. Maltoni and H.-S. Shao, NLO predictions for the production of a spin-two particle at the LHC, Phys. Lett. B 770 (2017) 507 [arXiv: 1605.09359] [INSPIRE].

[16] S. Kraml, U. Laa, K. Mawatari and K. Yamashita, Simplified dark matter models with a spin-2 mediator at the LHC, Eur. Phys. J. C 77 (2017) 326 [arXiv:1701.07008] [InSPIRE].

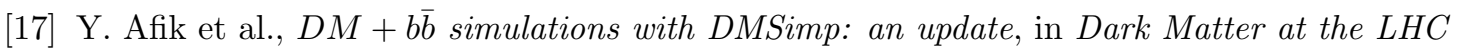
2018: Experimental and theoretical workshop (DM@LHC 2018), Heidelberg, Germany, April 3-6, 2018 (2018) [arXiv: 1811.08002] [INSPIRE].

[18] J. Alwall et al., The automated computation of tree-level and next-to-leading order differential cross sections and their matching to parton shower simulations, JHEP 07 (2014) 079 [arXiv: 1405.0301] [INSPIRE].

[19] A. Alloul, N.D. Christensen, C. Degrande, C. Duhr and B. Fuks, FeynRules 2.0 - A complete toolbox for tree-level phenomenology, Comput. Phys. Commun. 185 (2014) 2250 [arXiv: 1310.1921] [INSPIRE].

[20] C. Degrande, Automatic evaluation of UV and R2 terms for beyond the Standard Model Lagrangians: a proof-of-principle, Comput. Phys. Commun. 197 (2015) 239 [arXiv: 1406.3030] [INSPIRE].

[21] XENON collaboration, Dark Matter Search Results from a One Ton-Year Exposure of XENON1T, Phys. Rev. Lett. 121 (2018) 111302 [arXiv:1805.12562] [INSPIRE].

[22] M. Beneke and V.A. Smirnov, Asymptotic expansion of Feynman integrals near threshold, Nucl. Phys. B 522 (1998) 321 [hep-ph/9711391] [InSPIRE].

[23] D. de Florian, A. Kulesza and W. Vogelsang, Threshold resummation for high-transverse-momentum Higgs production at the LHC, JHEP 02 (2006) 047 [hep-ph/0511205] [INSPIRE].

[24] S. Catani, M.L. Mangano and P. Nason, Sudakov resummation for prompt photon production in hadron collisions, JHEP 07 (1998) 024 [hep-ph/9806484] [INSPIRE].

[25] S. Catani, M.L. Mangano, P. Nason, C. Oleari and W. Vogelsang, Sudakov resummation effects in prompt photon hadroproduction, JHEP 03 (1999) 025 [hep-ph/9903436] [INSPIRE].

[26] E. Laenen, G. Oderda and G.F. Sterman, Resummation of threshold corrections for single particle inclusive cross-sections, Phys. Lett. B 438 (1998) 173 [hep-ph/9806467] [InSPIRE]. 
[27] G.F. Sterman and W. Vogelsang, Threshold resummation and rapidity dependence, JHEP 02 (2001) 016 [hep-ph/0011289] [INSPIRE].

[28] D. de Florian and W. Vogelsang, Threshold resummation for the prompt-photon cross section revisited, Phys. Rev. D 72 (2005) 014014 [hep-ph/0506150] [INSPIRE].

[29] N. Kidonakis and J.F. Owens, Soft gluon resummation and NNLO corrections for direct photon production, Phys. Rev. D 61 (2000) 094004 [hep-ph/9912388] [INSPIRE].

[30] N. Kidonakis and V. Del Duca, Electroweak boson hadroproduction at large transverse momentum: Factorization, resummation and NNLO corrections, Phys. Lett. B 480 (2000) 87 [hep-ph/9911460] [INSPIRE].

[31] N. Kidonakis and A. Sabio Vera, W hadroproduction at large transverse momentum beyond next-to-leading order, JHEP 02 (2004) 027 [hep-ph/0311266] [INSPIRE].

[32] R.J. Gonsalves, N. Kidonakis and A. Sabio Vera, $W$ production at large transverse momentum at the large hadron collider, Phys. Rev. Lett. 95 (2005) 222001 [hep-ph/0507317] [INSPIRE].

[33] S. Catani, M. Grazzini and A. Torre, Soft-gluon resummation for single-particle inclusive hadroproduction at high transverse momentum, Nucl. Phys. B 874 (2013) 720 [arXiv: 1305.3870] [INSPIRE].

[34] P. Hinderer, F. Ringer, G. Sterman and W. Vogelsang, Threshold Resummation at NNLL for Single-particle Production in Hadronic Collisions, Phys. Rev. D 99 (2019) 054019 [arXiv: 1812.00915] [INSPIRE].

[35] A. Bacchetta, G. Bozzi, M. Lambertsen, F. Piacenza, J. Steiglechner and W. Vogelsang, Difficulties in the description of Drell-Yan processes at moderate invariant mass and high transverse momentum, arXiv:1901.06916 [INSPIRE].

[36] T. Becher and M.D. Schwartz, Direct photon production with effective field theory, JHEP 02 (2010) 040 [arXiv:0911.0681] [INSPIRE].

[37] T. Becher, C. Lorentzen and M.D. Schwartz, Resummation for $W$ and $Z$ production at large pT, Phys. Rev. Lett. 108 (2012) 012001 [arXiv:1106.4310] [INSPIRE].

[38] F.P. Huang, C.S. Li, H.T. Li and J. Wang, Renormalization-group improved predictions for Higgs boson production at large $p_{T}$, Phys. Rev. D 90 (2014) 094024 [arXiv:1406.2591] [INSPIRE].

[39] M. Bonvini, S. Forte, M. Ghezzi and G. Ridolfi, Threshold Resummation in SCET vs. Perturbative QCD: An Analytic Comparison, Nucl. Phys. B 861 (2012) 337 [arXiv: 1201.6364] [INSPIRE].

[40] G. Sterman and M. Zeng, Quantifying Comparisons of Threshold Resummations, JHEP 05 (2014) 132 [arXiv:1312.5397] [INSPIRE].

[41] M. Bonvini, S. Forte, G. Ridolfi and L. Rottoli, Resummation prescriptions and ambiguities in SCET vs. direct QCD: Higgs production as a case study, JHEP 01 (2015) 046 [arXiv: 1409.0864] [INSPIRE].

[42] M. Beneke, P. Falgari, J. Piclum, C. Schwinn and C. Wever, Higher-order soft and Coulomb corrections to squark and gluino production at the LHC, PoS (RADCOR2013) 051 (2013) [arXiv: 1312.0837] [INSPIRE]. 
[43] A. Kulesza, L. Motyka, T. Stebel and V. Theeuwes, Associated $t \bar{t} H$ production at the LHC: Theoretical predictions at NLO+NNLL accuracy, Phys. Rev. D 97 (2018) 114007 [arXiv: 1704.03363] [INSPIRE].

[44] W. Beenakker, C. Borschensky, M. Krämer, A. Kulesza and E. Laenen, NNLL-fast: predictions for coloured supersymmetric particle production at the LHC with threshold and Coulomb resummation, JHEP 12 (2016) 133 [arXiv:1607.07741] [INSPIRE].

[45] S. Catani, M.L. Mangano, P. Nason and L. Trentadue, The Resummation of soft gluons in hadronic collisions, Nucl. Phys. B 478 (1996) 273 [hep-ph/9604351] [INSPIRE].

[46] A. Kulesza, G.F. Sterman and W. Vogelsang, Joint resummation in electroweak boson production, Phys. Rev. D 66 (2002) 014011 [hep-ph/0202251] [INSPIRE].

[47] http://feynrules.irmp.ucl.ac.be/wiki/DMsimp.

[48] J. Butterworth et al., PDF4LHC recommendations for LHC Run II, J. Phys. G 43 (2016) 023001 [arXiv: 1510.03865 ] [INSPIRE].

[49] S. Dulat et al., New parton distribution functions from a global analysis of quantum chromodynamics, Phys. Rev. D 93 (2016) 033006 [arXiv: 1506.07443] [InSPIRE].

[50] L.A. Harland-Lang, A.D. Martin, P. Motylinski and R.S. Thorne, Parton distributions in the LHC era: MMHT 2014 PDFs, Eur. Phys. J. C 75 (2015) 204 [arXiv: 1412.3989] [INSPIRE].

[51] NNPDF collaboration, Parton distributions for the LHC Run II, JHEP 04 (2015) 040 [arXiv: 1410.8849] [INSPIRE].

[52] A. Buckley et al., LHAPDF6: parton density access in the LHC precision era, Eur. Phys. J. C 75 (2015) 132 [arXiv: 1412.7420] [INSPIRE]. 